\title{
Tomasch effect in nanoscale superconductors
}

\author{
L.-F. Zhang, ${ }^{*}$ L. Covaci, ${ }^{\dagger}$ and F. M. Peeters ${ }^{\ddagger}$ \\ Departement Fysica, Universiteit Antwerpen, Groenenborgerlaan 171, B-2020 Antwerpen, Belgium
}

(Received 19 September 2014; published 16 January 2015)

\begin{abstract}
The Tomasch effect (TE) is due to quasiparticle interference (QPI) as induced by a nonuniform superconducting order parameter, which results in oscillations in the density of states (DOS) at energies above the superconducting gap. Quantum confinement in nanoscale superconductors leads to an inhomogenerous distribution of the Cooperpair condensate, which, as we found, triggers the manifestation of a new TE. We investigate the electronic structure of nanoscale superconductors by solving the Bogoliubov-de Gennes (BdG) equations self-consistently and describe the TE determined by two types of processes, involving two- or three-subband QPIs. Both types of QPIs result in additional BCS-like Bogoliubov-quasiparticles and BCS-like energy gaps leading to oscillations in the DOS and modulated wave patterns in the local density of states. These effects are strongly related to the symmetries of the system. A reduced $4 \times 4$ inter-subband BdG Hamiltonian is established in order to describe analytically the TE of two-subband QPIs. Our study is relevant to nanoscale superconductors, either nanowires or thin films, Bose-Einsten condensates, and confined systems such as two-dimensional electron gas interface superconductivity.
\end{abstract}

DOI: 10.1103/PhysRevB.91.024508

PACS number(s): 74.78.Na, 74.81.-g, 74.20.Pq

\section{INTRODUCTION}

Electronic structure has always been one of the most important topics in understanding transport properties in condensed matter. A variety of remarkable phenomena, from traditional conducting, semiconducting or insulating behavior to contemporary quantum Hall effect [1] and topological insulator behavior [2], are induced by a diversity of electronic structures.

For conventional superconductors, the well-known BCS theory [4] and its generalization, the Bogoliubov-de Gennes $(\mathrm{BdG})$ equations [3], are the milestones needed to reveal the electronic structure theoretically. An energy gap created around the Fermi level, $E_{F}$, in homogeneous superconductivity and low-lying bound states existing in the core of vortex states [5-11] were successfully predicted and coincide with experimental results from tunneling conductance and scanning tunneling microscopy (STM) [12-15].

Nanoscale superconductors have also received considerable attention in the last decades due to developments in nanotechnology and their unique properties such as shell effect [16], quantum-size effect [17-21], and quantum-size cascades under magnetic field [22]. All these effects are induced by changes in the electronic structure resulting from quantum confinement, such that energy levels are discretized in nanoparticles and single-electron subbands appears in nanowires. In addition, such electronic structure results in a spatially inhomogeneous superconducting order parameter [20], which further induces other effects such as superconducting multigap structures [23,24], new Andreev states [24], unconventional vortex states [25], and a position-dependent impurity effect [26].

An interesting phenomenon that appears due to inhomogeneous superconductivity is the Tomasch effect (TE), which is a consequence of quasiparticle interference (QPI)

\footnotetext{
*lingfeng.zhang@uantwerpen.be

†lucian@covaci.org

†francois.peeters@uantwerpen.be
}

due to scattering on a nonuniform energy gap [27-30]. The underlying process consists in a quasiparticle interacting with, and being condensed into, the sea of Cooper pairs leaving behind a different but degenerate quasiparticle [29,30]. As a result, the Tomasch oscillations appear as periodic oscillations in the density of states (DOS) in superconducting junctions, at energies larger than the superconducting gap [10,27,28,31]. These oscillations could be further enhanced when considering layered structures formed by intercalating successive metallic and superconducting regions. In this case, the order parameter is by design nonhomogeneous. Theoretically, these oscillations were studied in multilayer structures by using Green's functions methods like Gor'kov equations [32,33]. However, the electronic structure under TE has not been unveiled because a fully self-consistent numerical calculation is required in order to obtain the coherence between quasiparticle states.

QPIs should also be observed in unconventional superconductors [34-36], where the interference should be more pronounced due to the intrinsic inhomogeneous nature of the superconducting order parameter. Theoretically, QPI due to a local superconducting order parameter suppression in large unconventional samples was demonstrated in Ref. [37]. However, in nanoscale structures, quantum confinement modifies the symmetries of the electronic states. Thus the signatures of QPI in nanoscale superconductors, although of same nature, could be different in manifestation in large samples but with intrinsic inhomogeneities [37].

In this paper, we investigate the electronic structure of clean nanoscale superconductors by solving the Bogoliubov-de Gennes (BdG) equations self-consistently. We focus on the TE, which appears above the superconducting gap. High-precision energy excitation spectra are needed in order to see the effect of the QPI processes clearly. Two geometries, nanobelts and nanowires, are used as typical examples in order to unveil the properties of TE resulting from two- and three-subband QPIs. The importance of the sample symmetry is discussed. We find that even in the presence of weak disorder, the Tomasch effect is robust. 
It is important to keep in mind that the mean-field BdG approach has limited validity when describing superconducting nanobelts and nanowires with diameters down to $10 \mathrm{~nm}$ [38-41]. For such nanoscale samples, fluctuations might play an important role, but are totally neglected by a mean-field method. Moreover, the quasiparticles in the Landau-Fermi liquid theory are only well defined near the Fermi level. Therefore the discussion and results presented in this paper are valid for larger samples where these effects are not significant.

The paper is organized as follows. In Sec. II, we first investigate TE of two-subband QPI in nanobelts. The twodimensional BdG equations are outlined for nanobelts in Sec. II A. Properties of the electronic structures under the TE of two-subband QPI are presented in Sec. II B. A description based on a reduced $4 \times 4 \mathrm{BdG}$ matrix is next introduced in Sec. II $C$ in order to explain the properties of TE as due to two-subband QPI. A possible observable effect, modulated wave patterns in LDOS, induced by TE of two-subband QPI is discussed in Sec. II D. Signatures of QPI under the influence of weak impurities are discussed in Sec. II E. Next, we investigate TE of three-subband QPI in nanowires in Sec. III, where the three-dimensional BdG equations are solved for nanowires in Sec. III A. The electronic structure under the influence of TE of three-subband QPI and their symmetry dependent properties are presented in Sec. III B. Finally, our conclusions are summarized in Sec. IV.

\section{TOMASCH EFFECT IN SUPERCONDUCTING NANOBELTS}

\section{A. Bogoliubov-de Gennes equations for two-dimensional nanobelts}

For a conventional superconductor in the clean limit, the BdG equations in the absence of a magnetic field can be written as

$$
\left(\begin{array}{cc}
H_{e} & \Delta(\vec{r}) \\
\Delta(\vec{r})^{*} & -H_{e}^{*}
\end{array}\right)\left(\begin{array}{l}
u_{n}(\vec{r}) \\
v_{n}(\vec{r})
\end{array}\right)=E_{n}\left(\begin{array}{c}
u_{n}(\vec{r}) \\
v_{n}(\vec{r})
\end{array}\right),
$$

where $H_{e}=-(\hbar \nabla)^{2} / 2 m-E_{F}$ is the single-electron Hamiltonian with $E_{F}$ the Fermi energy, $u_{n}\left(v_{n}\right)$ are electron(hole)-like quasiparticle eigen-wave-functions and $E_{n}$ are the quasiparticle eigenenergies. The $u_{n}\left(v_{n}\right)$ obey the normalization condition

$$
\int\left(\left|u_{n}(\vec{r})\right|^{2}+\left|v_{n}(\vec{r})\right|^{2}\right) d \vec{r}=1 .
$$

The superconducting order parameter is determined selfconsistently from the eigen-wave-functions and eigenenergies:

$$
\Delta(\vec{r})=g \sum_{E_{n}<E_{c}} u_{n}(\vec{r}) v_{n}^{*}(\vec{r})\left[1-2 f\left(E_{n}\right)\right],
$$

where $g$ is the coupling constant, $E_{c}$ is the cutoff energy, and $f\left(E_{n}\right)=\left[1+\exp \left(E_{n} / k_{B} T\right)\right]^{-1}$ is the Fermi distribution function, where $T$ is the temperature. The core part of Eq. (3) is the pair amplitude, which is defined as

$$
D_{n}(\vec{r})=u_{n}(\vec{r}) v_{n}^{*}(\vec{r}) .
$$

The pair amplitude is the key parameter that shows the coupling between electronlike and holelike quasiparticles for each Bogoliubov quasiparticle.
In this section, we consider a two-dimensional nanobelt. The width is $W$ in the transverse direction, $x$, and, because of confinement, Dirichlet boundary conditions are used at the surface (i.e., $\left.u_{n}\right|_{x=0}=\left.u_{n}\right|_{x=W}=0,\left.v_{n}\right|_{x=0}=\left.v_{n}\right|_{x=W}=$ 0 ). We consider periodic boundary conditions along the $y$ direction, with a unit cell of length $L$. The length is set to be large enough in order to guarantee that physical properties are $L$ independent.

In order to solve the BdG equations (1)-(3) numerically, we expand $u_{n}\left(v_{n}\right)$ as

$$
\left(\begin{array}{l}
u_{n}(\vec{r}) \\
v_{n}(\vec{r})
\end{array}\right)=\sum_{j \in \mathbb{N}^{+}, k} \varphi_{j k}(x, y)\left(\begin{array}{c}
u_{j k}^{n} \\
v_{j k}^{n}
\end{array}\right),
$$

where

$$
\varphi_{j k}(x, y)=\sqrt{\frac{2}{W}} \sin \left(\frac{\pi j x}{W}\right) \frac{e^{i k y}}{\sqrt{L}}
$$

are the eigenstates of the single-electron Schrödinger equation $H_{e} \phi_{j k}=\zeta_{j k} \phi_{j k}$ where the wave vector $k=2 \pi m / L, m \in \mathbb{Z}$. The expansion in Eq. (5) has to include all the states with energy in the range $-E_{F}<\zeta_{j k}<E_{F}+\epsilon$ in order to allow the emergence of the TE well above the energy gap. The energy $\epsilon$ is taken to be $5 E_{c}$, which guarantees sufficient accuracy. We checked that higher $\epsilon$ does not change the results.

We remark that, for the chosen geometry, the order parameter depends only on the transverse variable $x$, i.e., $\Delta(\vec{r})=$ $\Delta(x)$. This implies no net momentum of the condensate in the $y$ direction and the quasiparticle amplitudes $\left(u_{n}, v_{n}\right)^{T}$ are $k$-separated. Then, the summation over $k$ in Eq. (5) can be removed and the BdG equation (1) is converted into a matrix equation for each $k$ whose contribution to $\Delta$ can be calculated independently from the other values of $k$. This allows us to include millions of quasiparticle states allowing very high resolution in the energy dispersion, which is necessary to observe clearly the Tomasch effect.

The local density of states (LDOS) is calculated as usual:

$$
A(\vec{r}, E)=\sum_{n}\left[\left|u_{n}(\vec{r})\right|^{2} \delta\left(E-E_{n}\right)+\left|v_{n}(\vec{r})\right|^{2} \delta\left(E+E_{n}\right)\right],
$$

and the total density of states (DOS) is obtained as

$$
N(E)=\int A(\vec{r}, E) d \vec{r} .
$$

The spectral weight is

$$
Z_{n}=\int\left|u_{n}(\vec{r})\right|^{2} d \vec{r},
$$

which represents the contribution of the electronic part of the wave function of a Bogoliubov quasiparticle state.

In this section, we set the microscopic parameters to be the same as those used in Refs. [7,10]. These parameters for bulk are the following: effective mass $m=2 m_{e}, E_{F}=40 \mathrm{meV}$, $E_{c}=3 \mathrm{meV}$ and coupling constant $g$ is set so that the bulk gap at zero temperature is $\Delta_{0}=1.2 \mathrm{meV}$, which yields $T_{c} \approx$ $8.22 \mathrm{~K}, \xi_{0}=\hbar v_{F} /\left(\pi \Delta_{0}\right)=14.7 \mathrm{~nm}$, and $k_{F} \xi_{0}=21.23$. The prototype material can be, e.g., $\mathrm{NbSe}_{2}[7,10]$. For nanobelts, the mean electron density $n_{e}$ is kept to the value obtained when $W, L \rightarrow \infty$ by using an effective $E_{F}$, where $n_{e}=$ $\frac{2}{S} \sum_{n} \int\left\{\left|u_{n}\right|^{2} f\left(E_{n}\right)+\left|v_{n}\right|^{2}\left[1-f\left(E_{n}\right)\right]\right\} d \vec{r}$ and $S=W L$ is 

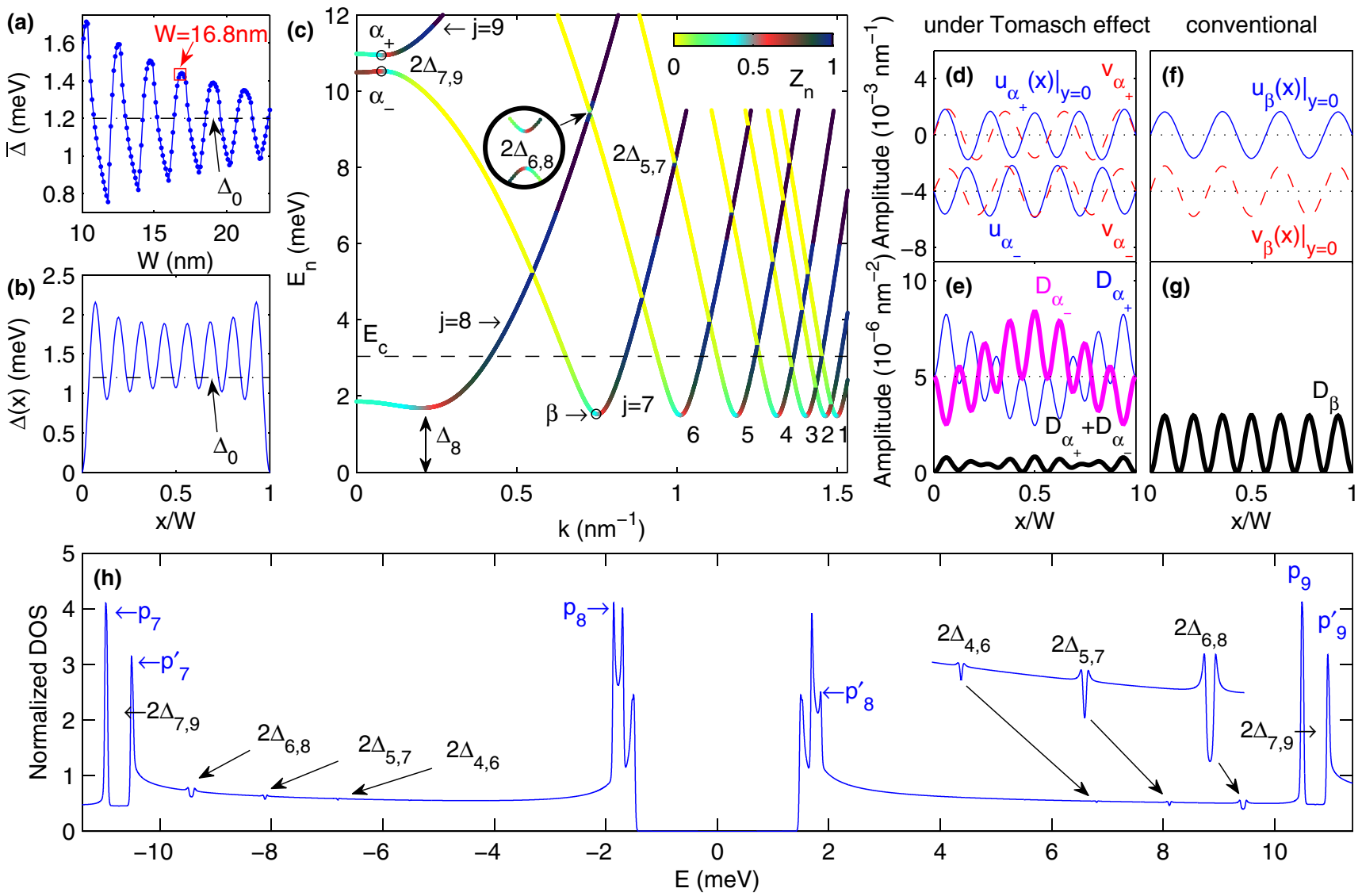

FIG. 1. (Color online) Nanobelt in the clean limit. (a) Spatially averaged $\bar{\Delta}$ as a function of width $W$. In the following we take, $W=16.8 \mathrm{~nm}$ (marked by an open square), which is in the resonant regime. (b) Order parameter, $|\Delta(x)|$, as a function of $x$. The horizontal dashed line indicates the bulk value of the order parameter, $\Delta_{0}$, in (a) and (b). (c) Quasiparticle excitation spectrum as a function of positive longitudinal wave vector $k$ for the relevant single-electron subband $j$. The spectral weight of the states is indicated by color. The horizontal dashed line indicates the cutoff energy $E_{c} . \Delta_{j}$ is the superconducting energy gap for states of subband $j$ and $2 \Delta_{j j^{\prime}}$ is the energy gap between the states of subbands $j$ and $j^{\prime}$ appearing due to the Tomasch effect. The electron and hole amplitudes, $u$ and $v$, (at $y=0$ as a function of $x$ ) of states $\alpha_{+}$ and $\alpha_{-}$[see (c)], which are under the influence of the Tomasch effect, are shown in (d). Their pair amplitudes, $D_{\alpha_{+}}$and $D_{\alpha_{-}}$, and the total one, $D_{\alpha_{+}}+D_{\alpha_{-}}$, are shown in (e). Note that $D_{\alpha_{+}}$and $D_{\alpha_{-}}$are shifted up for clarity. In (f) and (g), we show $u, v$, and $D$ as a function of $x$ for the state $\beta$ [see (c)], respectively. (h) The corresponding density of states. Peaks $p_{j}$ are due to the contributions from the states of the bottom of the subbands $j$, while accompanying peaks $p^{\prime}{ }_{j}$ are due to the formation of the gaps, i.e., electron-hole coupling.

the area of the unit cell. All the calculations are performed at zero temperature.

\section{B. Tomasch effect from two-subband quasiparticle interference}

Results for the nanobelt with width $W=16.8 \mathrm{~nm}$ are presented in Fig. 1. We first discuss its general properties, which will be used as a reference later on when the system is under the influence of TE.

The width of the nanobelt is about $\xi_{0}$ such that the quantum size effect is significant. The spatially averaged order parameter $\bar{\Delta}$ is shown in Fig. 1(a) as a function of the width $W$. This nanobelt with width $W=16.8 \mathrm{~nm}$ is at resonance and $\bar{\Delta}$ is about $20 \%$ higher than the bulk one, $\Delta_{0}$. As seen from Fig. 1(b), the spatial distribution of the order parameter, $\Delta(x)$, is clearly enhanced for most of the $x$ values and shows strong wavelike oscillations. This enhancement is induced by quasiparticle states from the bottom of subband $j=8$ which have a large spectral weight as seen from Fig. 1(c). For this narrow nanobelt, only a few subbands $(j=1-8)$ contribute to the order parameter and are distinguishable from each other in Fig. 1(c).

For a superconductor under quantum confinement and thus with inhomogeneous order parameter, a multigap structure was predicted $[23,24]$. The energy gap $\Delta_{j}$ of the subband $j$ is defined by the lowest energy of that subband. As seen from Fig. 1(c), the energy gaps $\Delta_{1}, \ldots, \Delta_{7}$ have almost the same value but are lower than $\Delta_{8}$. This feature can be seen clearly in the corresponding DOS, in Fig. 1(h), where three significant pairs of coherence peaks form around the Fermi level. The lowest energy pair of peaks are less important and are due to the contributions of quasiparticles of subbands $j=1-7$. The second lowest pair of peaks are more significant and most of the contributions are given by quasiparticle states of the subband $j=8$ around $k=0.2 \mathrm{~nm}^{-1}$ where it reaches its local minimum, as shown in Fig. 1(c). It is worth noting that these two pairs of peaks show electron-hole symmetry in the DOS, whereas the third lowest pair of peaks do not. The 
latter ones are due to the contribution from states of subband $j=8$ around $k=0$. The more significant peak in negative bias is due to: (1) a large number of hole quasiparticle states at the bottom of subband $j=8$, and (2) the electron-hole coupling asymmetry due to the higher energy level where the Bogoliubov quasiparticle states are formed by the larger weight of the hole component. This can be seen from Fig. 1(c) where the spectral weight $Z_{n}$ represents the color(shade) of the lines.

In an isotropic superconductor, Bogoliubov quasiparticles are well defined only for energies close to the superconducting gap $\Delta$. For such states, the electron and hole components are of the same weight, which maximizes the amplitude of the pair amplitudes that generate the order parameter. With increasing energy, Bogoliubov quasiparticles decompose into dominant electron and hole components, accompanied by a dramatically decreasing pair amplitude. Finally, they decompose into separate electron or hole quasiparticles belonging to the normal state.

For a conventional Bogoliubov quasiparticle, which is well formed for energies near the gap, the electron and hole components belong always to the same subbands $j$, i.e., $j-j$ coupling. As an example, we show more details of the lowest energy state $\beta$ of subband $j=7$ [marked by an open circle in Fig. 1(c)] in Figs. 1(f) and 1(g). The electron and hole amplitudes at $y=0$, i.e., $\left.u_{\beta}\right|_{y=0}$ and $\left.v_{\beta}\right|_{y=0}$, as a function of $x$ are shown in Fig. 1(f). $v_{\beta}$ are shifted down for clarity. It can be seen that both components are in phase because they belong to the same subband $j=7$. Thus the pair amplitude $D_{\beta}$ shown in Fig. $1(\mathrm{~g})$ as a function of $x$, is always positive and shows a regular wavelike pattern with a constant envelope.

We next discuss the influence of the TE on the electronic structure. First, we find that energy gaps are unexpectedly opened between electron and hole quasiparticle states even well above the Fermi level. As seen from Fig. 1(c), the most pronounced energy gap above $E_{c}$ is generated by states $\alpha_{+}$ and $\alpha_{-}$(marked by open circles) where subbands $j=7$ and 9 were supposed to cross each other in case of a homogeneous superconductor. Here, the states $\alpha_{ \pm}$have the same $k$ value and they are chosen because they have the minimal gap $2 \Delta_{7,9}$ between the two subbands, i.e., $E_{\alpha_{+}}-E_{\alpha_{-}}=2 \Delta_{7,9}$. The energy of state $\alpha_{+}$is higher than the state of $\alpha_{-}$. As there are only two quasiparticles that take part in the QPI, we refer to this effect as the Tomasch effect determined by two-subband quasiparticles interference processes.

Second, we find that particle-hole mixing (Bogoliubov quasiparticles) is always significant for states under the influence of the TE. This can be seen from the spectral weight of the related quasiparticle states in Fig. 1(c). Their color changes gradually for both the upper and lower energy bands that are associated with this gap. Furthermore, the electron and hole wave-functions of the states under the TE have a different structure because they belong to different subbands, $j$ and $j^{\prime}$, i.e., $j-j^{\prime}$ coupling. To see this characteristic, we show electron and hole amplitudes at $y=0,\left.u\right|_{y=0}$ and $\left.v\right|_{y=0}$, of the states $\alpha_{ \pm}$as a function of $x$ in Fig. 1(d). Note that the amplitudes of the state $\alpha_{-}$are shifted down for clarity. For both states, $\alpha_{ \pm}$, the spectral weights of the electron components are the same as those of the hole components, which resembles the conventional Bogoliubov quasiparticle state $\beta$. However, their electron and hole wave-functions show a phase shift because the electron components, $u_{\alpha_{ \pm}}$, belong to subband $j=9$ and the hole component, $v_{\alpha_{ \pm}}$, to subband $j=7$. This can be noticed by counting the numbers of nodes of the wave functions in Fig. 1(d). The difference between these states is that $\alpha_{+}$ is the bonding(symmetric) combination of the electron and hole components while $\alpha_{-}$is the antibonding(antisymmetric) combination, i.e., $\left|\alpha_{ \pm}\right\rangle= \pm\left|u_{j=9}\right\rangle+\left|v_{j=7}\right\rangle$, as seen from Fig. 1(d). As a result, their pair amplitudes $D_{\alpha_{ \pm}}$as a function of $x$, shown in Fig. 1(e) are not positive-definite and exhibit a phase shift in space.

Third, the Bogoliubov quasiparticle states under the influence of TE do not directly affect the order parameter. As seen from Fig. 1(e), the pair amplitudes $D_{\alpha_{ \pm}}$are in antiphase due to the nature of bonding and antibonding combinations. Thus, their net pair amplitude $D_{\text {net }}=D_{\alpha_{+}}+D_{\alpha_{-}}$almost cancels out as shown in Fig. 1(e). It is worth noting that, as proven in the next section, the net pair amplitude of these states have nothing to do with the TE. This contribution is always positive, while the superconducting order parameter is weakly affected by TE even if the energy of this avoided crossing is below the cutoff energy $E_{c}$.

Fourth, TE results in BCS-like pseudogaps in the DOS, which are symmetrically located on both positive and negative bias. This is easy to understand because energy gaps are opened for the relevant crossing subbands where particle-hole mixing appears under the influence of the TE. Typically, the gaps induced by the TE are smaller than the main superconducting gap. They will appear as pseudo-gaps because of the underlying background given by the other subbands, which are not gapped. For example, as shown in Fig. 1(h), the largest gap induced by TE, $2 \Delta_{7,9}$ is only about $0.42 \mathrm{meV}$. However, the effect of the gap resulting from the TE can be seen more clearly from the enhanced peaks appearing at the gap edge when the bottom of one subband touches the top of the other subband. In Fig. 1(h), the gap $2 \Delta_{7,9}$ is surrounded by the peaks $p_{j=7}$ and $p_{j=7}^{\prime}$ in DOS for negative bias and by $p_{j=9}$ and $p_{j=9}^{\prime}$ for positive bias. The generation of the two pair of peaks are similar to the $p_{j=8}, p_{j=8}^{\prime}$ except that they result from the top of the holelike subband $j=7$ and from the bottom of the electronlike subband $j=9$.

For the chosen width, there are more gap structures induced by $\mathrm{TE}$ in the excitation spectrum [see Fig. 1(c)] and corresponding peaks in the DOS [see Fig. 1(h)]. For example, the gap $2 \Delta_{6,8}$ appears for the coupling of states from subbands $j=6$ and 8 at $E=9.4 \mathrm{meV}$ but its influence on the DOS is weak. We also find other gaps such as $2 \Delta_{5,7}, 2 \Delta_{4,6}$ and $2 \Delta_{3,5}$.

TE is a common effect in inhomogeneous superconductivity and is strongly related to the symmetry, parity and structure of the order parameter. In the case of the clean limit, as we showed here, it is important to realize that avoided crossings exist only between electron and hole quasiparticle states of subbands $j$ and $j+2 n$, where $n$ is an integer. This is because the order parameter has an even function with respect to $y=0$. Similarly, an odd-functional order parameter would result in TE between states of subbands $j$ and $j+(2 n-1)$. In the arbitrary situation where the order parameter shows a random distribution due to strong disorder, TE should happen between all degenerate electron and hole quasiparticles. All 
these properties can be explained by a reduced $4 \times 4 \mathrm{BdG}$ matrix as shown in the next section.

\section{C. $4 \times 4$ BdG matrix for the two-subband quasiparticle interference}

Due to the fact that only two subbands are involved in the TE of two-subband QPI, we find it can be qualitatively described by a reduced $4 \times 4 \mathrm{BdG}$ matrix where only the two Bogoliubov quasiparticles and their correlations are considered. We start from the general BdG equations (1) but only keep a hole state of subband $j$ and an electron state of subband $j^{\prime}$ for a given wave vector $k$. For the hole state, its energy and wave function are determined by the single-electron Schrödinger equation $H_{e}|j\rangle=-\zeta_{j}|j\rangle$. Note that $-\zeta_{j}<0$ due to the hole excitation. Again, the energy and wave function of the electron state is determined by $H_{e}\left|j^{\prime}\right\rangle=\zeta_{j^{\prime}}\left|j^{\prime}\right\rangle$ where $\zeta_{j^{\prime}}>0$. The orthogonal relation between the hole state $|j\rangle$ and the electron state $\left|j^{\prime}\right\rangle$ yields $\left\langle j \mid j^{\prime}\right\rangle=\delta_{j j^{\prime}}$. For simplicity and fitting the case of previous section, $|j\rangle$ and $\left|j^{\prime}\right\rangle$ are chosen as real and to generate the real order parameter $\Delta(\vec{r})$. Then, the electron component of a Bogoliubov quasiparticle is $u_{n}=U_{j}|j\rangle+U_{j^{\prime}}\left|j^{\prime}\right\rangle$ and the hole component is $v_{n}=V_{j}|j\rangle+V_{j^{\prime}}\left|j^{\prime}\right\rangle$, where $U_{j}$ and $V_{j}$ are the component amplitude of subband $j$ for electron and hole, respectively. The $4 \times 4 \mathrm{BdG}$ matrix reads

$$
\left[\begin{array}{cccc}
-\zeta_{j} & 0 & \Delta_{j} & \Delta_{j j^{\prime}} \\
0 & \zeta_{j^{\prime}} & \Delta_{j^{\prime} j} & \Delta_{j^{\prime}} \\
\Delta_{j} & \Delta_{j j^{\prime}} & \zeta_{j} & 0 \\
\Delta_{j^{\prime} j} & \Delta_{j^{\prime}} & 0 & -\zeta_{j^{\prime}}
\end{array}\right]\left[\begin{array}{c}
U_{j}^{n} \\
U_{j^{\prime}}^{n} \\
V_{j}^{n} \\
V_{j^{\prime}}^{n}
\end{array}\right]=E_{n}\left[\begin{array}{c}
U_{j}^{n} \\
U_{j^{\prime}}^{n} \\
V_{j}^{n} \\
V_{j^{\prime}}^{n}
\end{array}\right]
$$

with the matrix elements $\Delta_{j}=\langle j|\Delta(\vec{r})| j\rangle$ and $\Delta_{j j^{\prime}}=$ $\left\langle j|\Delta(\vec{r})| j^{\prime}\right\rangle=\Delta_{j^{\prime} j}$. Note that the $\Delta_{j j^{\prime}}$ is the exchange integral between the two states from different subbands. In a homogeneous superconductor, the constant order parameter $\Delta(\vec{r}) \equiv \Delta$ leading to the zero exchange integral, $\Delta_{j j^{\prime}}=0$, results in the decomposition of Eq. (10) into two sets of general $2 \times 2 \mathrm{BdG}$ matrices for the two states respectively. Thus there is no TE for this case.

For an inhomogeneous superconductor with a perturbation in the order parameter $\Delta(\vec{r})=\Delta+\delta \Delta(\vec{r})$, the matrix elements are

$$
\Delta_{j}=\langle j|\Delta+\delta \Delta(\vec{r})| j\rangle \approx \Delta,
$$

and

$$
\Delta_{j j^{\prime}}=\left\langle j|\Delta+\delta \Delta(\vec{r})| j^{\prime}\right\rangle=\left\langle j|\delta \Delta(\vec{r})| j^{\prime}\right\rangle=\Delta_{j^{\prime} j} \neq 0 .
$$

Note that the nonzero exchange integral $\Delta_{j j^{\prime}} \neq 0$ in this case is responsible for the TE.

The TE of QPI reaches its maximum when states of two subbands are degenerate in energy, i.e., $\zeta_{j}=\zeta_{j^{\prime}}=\zeta$. So Eq. (10) is written as

$$
\left[\begin{array}{cccc}
-\zeta & 0 & \Delta & \Delta_{j j^{\prime}} \\
0 & \zeta & \Delta_{j j^{\prime}} & \Delta \\
\Delta & \Delta_{j j^{\prime}} & \zeta & 0 \\
\Delta_{j j^{\prime}} & \Delta & 0 & -\zeta
\end{array}\right]\left[\begin{array}{c}
U_{j}^{n} \\
U_{j^{\prime}}^{n} \\
V_{j}^{n} \\
V_{j^{\prime}}^{n}
\end{array}\right]=E_{n}\left[\begin{array}{c}
U_{j}^{n} \\
U_{j^{\prime}}^{n} \\
V_{j}^{n} \\
V_{j^{\prime}}^{n}
\end{array}\right] .
$$

The eigenvalues and eigenstates of matrix (13) are exactly solvable and the four eigenvalues are

$$
\pm E_{ \pm}= \pm \varepsilon \pm \Delta_{j j^{\prime}}
$$

where $\varepsilon=\sqrt{\zeta^{2}+\Delta^{2}}$ is the quasiparticle excitation energy of the isotropic superconducting gap $\Delta$. The gap induced by $\mathrm{TE}, \Delta_{T E}$, is the energy difference between the two positive eigenvalues:

$$
\Delta_{T E}=\left\{\begin{array}{ll}
2 \Delta_{j j^{\prime}} & \text { if } \Delta_{j j^{\prime}}<\varepsilon \\
2 \varepsilon & \text { if } \Delta_{j j^{\prime}} \geqslant \varepsilon
\end{array} .\right.
$$

Typically, the exchange integral $\Delta_{j j^{\prime}}$ is smaller than the excitation energy gap $\varepsilon$. As a result, $\Delta_{T E}=2 \Delta_{j j^{\prime}}$ and that is why we labeled the gaps as $2 \Delta_{j, j^{\prime}}$ in Fig. 1.

When the exchange integral is positive, i.e., $0<$ $\Delta_{j j^{\prime}}<\varepsilon$, the eigenvalues sorted by their values are $\left(\begin{array}{llll}-E_{+} & -E_{-} & E_{-} & E_{+}\end{array}\right)$and their corresponding eigenstates are

$$
\left(\begin{array}{c}
U_{j}^{n} \\
U_{j^{\prime}}^{n} \\
V_{j}^{n} \\
V_{j^{\prime}}^{n}
\end{array}\right)=\left(\begin{array}{rrrr}
A & A & B & B \\
B & -B & -A & A \\
-B & -B & A & A \\
-A & A & -B & B
\end{array}\right),
$$

where

$$
\begin{aligned}
& A=\frac{1}{2}\left(1+\frac{\zeta}{\varepsilon}\right)^{\frac{1}{2}}, \\
& B=\frac{1}{2}\left(1-\frac{\zeta}{\varepsilon}\right)^{\frac{1}{2}} .
\end{aligned}
$$

Normalization is chosen to satisfy Eq. (2), i.e., $2\left(A^{2}+B^{2}\right)=$ 1 . For both eigenstates with positive eigenvalue $E_{+}$and $E_{-}$, their spectral weights $Z_{E_{ \pm}}=0.5$ indicate that Bogoliubov quasiparticles are well formed by the coupling between the electron and hole subbands. The difference between the two states are the bonding and antibonding combinations of the electron and hole components.

It is interesting to realize that the $A$ and $B$ are $\Delta_{j j^{\prime}}$ independent and $\sqrt{2}(A B)^{T}$ is the eigenstate of the positive eigenvalue of the general $2 \times 2 \mathrm{BdG}$ equations, i.e.,

$$
\left(\begin{array}{cc}
\zeta & \Delta \\
\Delta & -\zeta
\end{array}\right)\left(\begin{array}{l}
\sqrt{2} A \\
\sqrt{2} B
\end{array}\right)=\varepsilon\left(\begin{array}{l}
\sqrt{2} A \\
\sqrt{2} B
\end{array}\right)
$$

where the eigenenergy is $\varepsilon$ and the $\sqrt{2}$ is introduced to satisfy the normalization condition, Eq. (2). It turns out that the total pair amplitude of the states $E_{+}$and $E_{-}$are the same as the one without TE, i.e.,

$$
D_{E_{+}}+D_{E_{-}}=2 A B\left(|j\rangle^{2}+\left|j^{\prime}\right\rangle^{2}\right)=D_{j}+D_{j^{\prime}}
$$

Finally, we have to mention that the exchange integral $\Delta_{j j^{\prime}}$ is sensitive to the symmetry, parity, and spatial variation of the order parameter $\Delta(\vec{r})$. For the nanobelt in the clean limit, $\Delta(\vec{r})$ has a spatial distribution with even parity with respect to $y=0$. The exchange integral is exactly zero when both states $|j\rangle$ and $\left|j^{\prime}\right\rangle$ have different parity. This is the reason why TE 


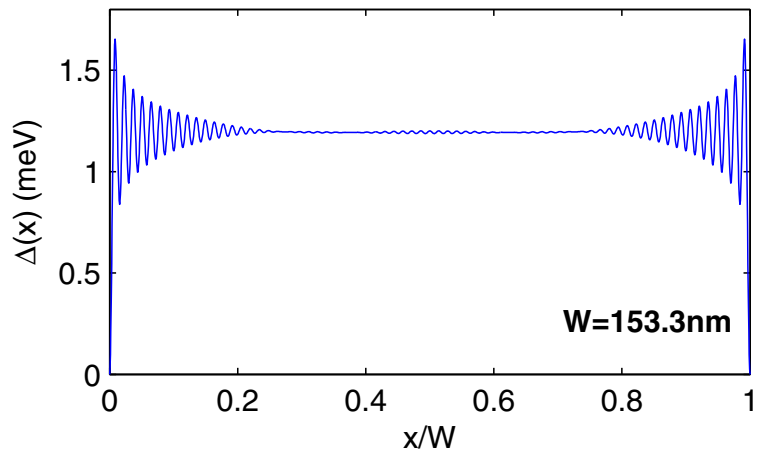

FIG. 2. (Color online) Spatial order parameter distribution $|\Delta(x)|$ for a sample with $W=153.3 \mathrm{~nm}$. Note that the order parameter converges to $\Delta_{0}$ in the bulk.

only appears between electron and hole quasiparticle states of subbands $j$ and $j+2 n$, which have the same parity, resulting in a possible nonzero exchange integral $\Delta_{j j^{\prime}}$.

\section{Modulated waves in the local density of states due to the Tomasch effect}

Previously, we introduced the properties of the TE of twosubband QPIs for a narrow sample. However, the mean-field BdG theory is of limited validity in such a case due to the increasing importance of phase fluctuations and, moreover, quasiparticles are not well defined far above the Fermi level. In this section, we investigate the TE in wider samples in order to avoid these issues. The results of this section show that (1) properties obtained previously are still valid, and (2) the TE results in a modulated wave structure in the local density of states, which should be observable in experiments.

As an example, we present results for a nanobelt with width $W=153.3 \mathrm{~nm}$, which is more than $10 \xi_{0}$. The spatial distribution of the order parameter $\Delta(x)$ is shown in Fig. 2. It can be seen that the order parameter shows Friedel-like oscillations at both edges but it converges to its bulk value $\Delta_{0}$ far away from the edges. The flat order parameter in the central area suppresses the TE. Fortunately, the energy gaps induced by TE can still be seen clearly in the corresponding quasiparticle excitation spectrum in the upper panel of Fig. 3. Here, we focus on the gaps at the intersection between states of subbands $j$ and $j+2$, which are indicated by a dashed curve. Due to the smaller energy difference between the adjacent subbands in the wider sample, the gaps appear at energies close to the superconducting gap energy $\Delta_{0}$ and far below the cutoff energy $E_{c}$, where the quasiparticles are well defined.

The TE exhibits all the properties that have been introduced previously except that the quasiparticle states generate considerable net pair amplitude, contributing to the order parameter. To show this feature, we present the electron and hole amplitudes, $u$ and $v$, and their pair amplitude, $D$, of selected quasiparticle states $\gamma_{+}$and $\gamma_{-}$(marked by open circle in the upper panel) as a function of $x$ in the lower panels of Fig. 3. Both states have the same wave vector $k$ and are separated by an energy gap due to the influence of TE. It can be seen that $u$ and $v$ exhibit rapid oscillations with slowly varying envelopes. The envelopes show modulated wave structures due to the combination of states of subbands $j$ and $j+2$. The difference in envelope for states $\gamma_{+}$and $\gamma_{-}$ are due to the bonding and antibonding combinations of the two single-electron wave functions, respectively. Meanwhile, the phase shift between the $u$ and $v$ components leads to more complex pattern in their pair amplitude. Finally, the net pair amplitude $D_{\gamma_{+}}+D_{\gamma_{-}}$(shown in the lower panel of Fig. 3) is large and positive, showing a modulated wave structure.

As a reference, we also present the electron amplitude, $u$, hole amplitude, $v$, and its pair amplitude, $D$, of a conventional Bogoliubov quasiparticle state $\gamma$ (also marked by open circle in the upper panel) as a function of $x$ in the lower panels of Fig. 3. Because $u$ and $v$ belong to the same subband $j$, they are in phase leading to a positive pair amplitude with a flat envelope.

The states $\gamma_{+}$and $\gamma_{-}$, which are under the influence of TE, induce peaks in the DOS and modulated wave structures in the LDOS. Figure 4 show the corresponding DOS in the lower panel and the LDOS under the influence of TE in the upper panel. In the DOS, the peaks induced by states $\gamma_{ \pm}$sit at the symmetrical bias energy $E= \pm 1.65 \mathrm{meV}$. The insets magnify the relevant areas. In the insets, the outer peaks are induced by the state $\gamma_{+}$while the inner peaks are induced by the state $\gamma_{-}$. The LDOS shows very different patterns at these two energies, which can be seen from the upper panels. For the outer peaks in DOS, the LDOS is enhanced at the edge whereas, for the inner peaks, it is enhanced at the center. The envelope of LDOS varies slowly as a function of $x$. Therefore it may be easily detected by STM.

\section{E. Discussion on the signature of the Tomasch effect in the presence of disorder}

Previously, only the TE in clean superconductors were considered. However, in all experiments, superconductors are rather "dirty," where additional scattering processes of quasiparticles appear due to surface roughness, impurities, substrate and so on. These factors will broaden the single-electron levels, modify electronic wave functions, reduce the electron mean free path and, thus, lead to noise and modifications in DOS and LDOS. In this section, we study TE under such additional scattering processes by introducing a random distribution of weak impurities. The results of this section show that (1) many more gaps induced by TE are opened in this case, (2) one can still recover the dominant gaps seen the clean limit by comparing the DOS of superconducting and normal states, and (3) the LDOS shows more complex scattering patterns but follows the same bonding and antibonding combination of involved quasiparticle wave functions.

The random impurities are introduced by using the potential $U(\vec{r})$ in the single-electron Hamiltonian defined Eq. (1), i.e., $H_{e}=-(\hbar \nabla)^{2} / 2 m+U(\vec{r})-E_{F}$. As a result, the order parameter, $\Delta(\vec{r})$, is no longer longitudinally independent, i.e., $\Delta(\vec{r})=\Delta(x, y)$. Following the numerical approach introduced in Sec. II A, we take a periodic unit cell with length $L$ and width $W$ and expand the electron(hole)-like quasiparticle wave functions $u_{n}\left(v_{n}\right)$ by using Eq. (6). Using Bloch's theorem, the $\mathrm{BdG}$ equations will be separated for each reciprocal vector. By considering a large number of $k$ points, we achieve a 


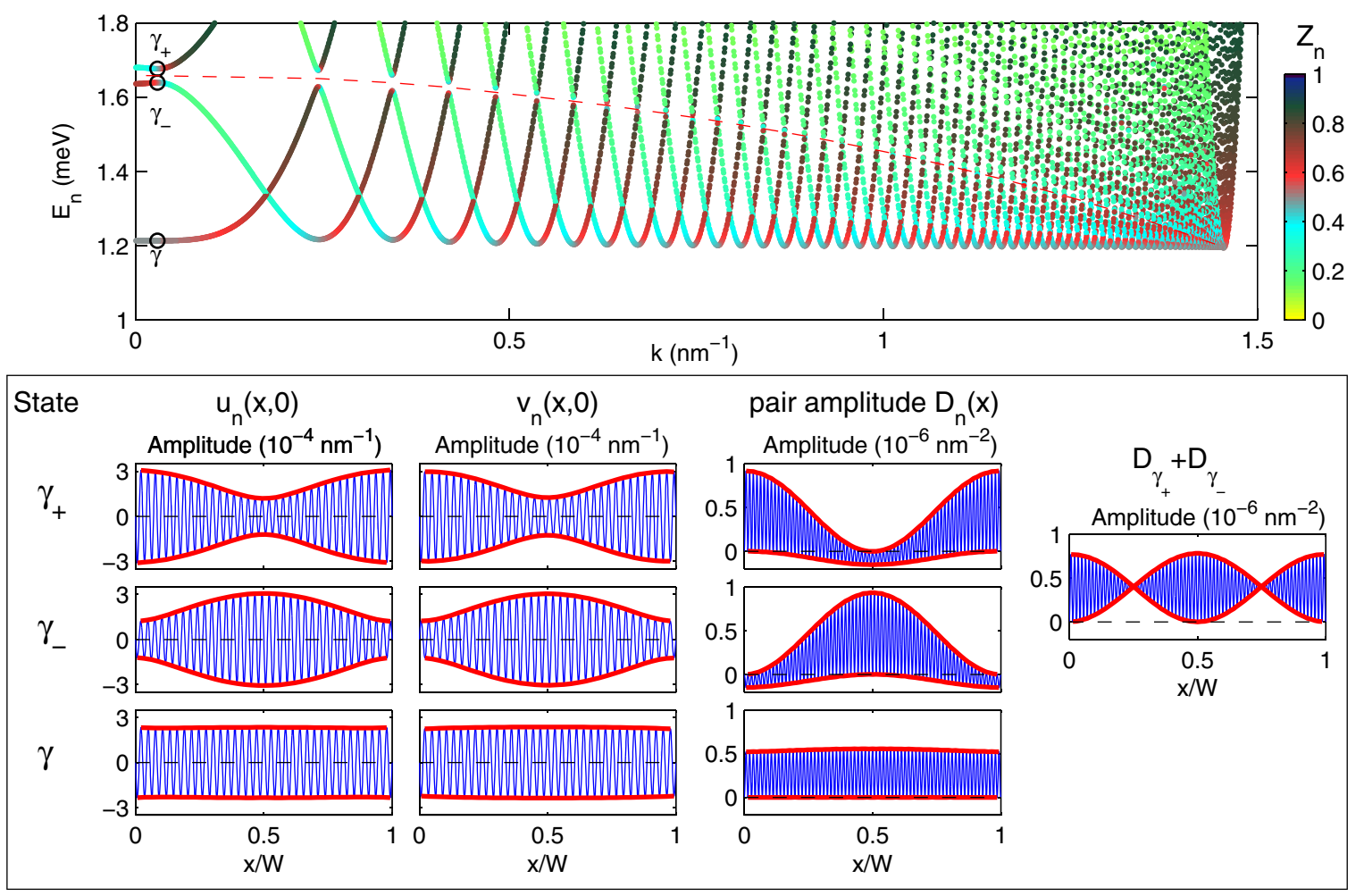

FIG. 3. (Color online) (Top) Quasiparticle excitation spectrum as a function of positive longitudinal wave vector, $k_{z}$, for energies below $1.8 \mathrm{meV}$ for a nanobelt of width $W=153.3 \mathrm{~nm}$. The spectral weight of the quasiparticle states are indicated by color. The three quasiparticle states $\gamma_{+}, \gamma_{-}$, and $\gamma$ marked by open circle are chosen for showing the electron and hole amplitudes, $u$ and $v$, as a function of $x$ at $y=0$ and their pair amplitudes $D$ in the lower panels. The red thick lines are the envelopes of the curves. The quasiparticle states $\gamma_{+}$and $\gamma_{-}$are under the influence of the Tomasch effect and show a phase difference between their $u$ and $v$ components. The net pair amplitude of the two states $D_{\gamma_{+}}+D_{\gamma_{-}}$is shown in the right-most panel.

good resolution in the DOS in order to observe the TE. In this section, we take $W=16.8 \mathrm{~nm}$ and $L=40 \mathrm{~nm}$. Such nanobelt in the clean limit $(U=0)$ has been introduced in Sec. II B. The impurity potential profile is modeled by a

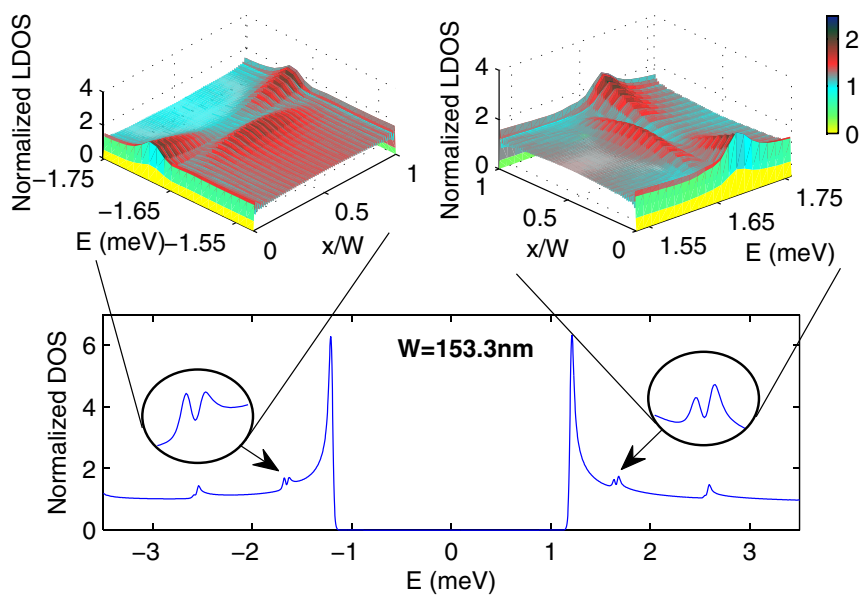

FIG. 4. (Color online) DOS and relevant LDOS for the sample with $W=153.3 \mathrm{~nm}$. The DOS is shown in the lower panel. The oscillatory structures induced by the Tomasch effect are magnified in the two insets. The corresponding $\operatorname{LDOS} A(x, E)$ along the transversal direction are shown in the upper panels. random collection of symmetric Gaussian functions, $U(\vec{r})=$ $\sum_{i} U_{i} \exp \left[-\left(\vec{r}-\vec{r}_{i}\right)^{2} / 2 \sigma^{2}\right]$, where $U_{i}$ is the amplitude, $\vec{r}_{i}$ is the location of the impurity and $\sigma=0.02$ represents the spread of the potential (full width at $1 / 10$ th of maximum is $0.86 \mathrm{~nm}$ ). For the situation of weak impurities (disorder), we take 100 impurities in the unit cell with random locations $r_{i}$ and random amplitude $U_{i}$ with a maximum $U_{i}^{\max }=0.1 E_{F}$. After the $\mathrm{BdG}$ equations are solved self-consistently, as described in the previous sections, the order parameter, $\Delta$, has almost the same distribution as the one with $U=0$.

We show in Fig. 5 the spectral weight of quasiparticles $Z_{n}$ as a function of energy of states $E_{n}$ for the case in the clean limit and the case with impurities. In clean bulk superconductors, particles and holes never mix at energies away from the superconducting gap, i.e., $Z_{n}=0$ or 1 , for holes or electrons, respectively. In the case of clean superconductor under quantum confinement, as seen from Fig. 5(a), particlehole mixing indicates the emergence of TE due to the stripelike inhomogeneity of the order parameter. In the presence of the impurities, as shown in Fig. 5(b), TE appears for much wider range of energies due to the symmetry broken of the electronic wave functions. It indicates that many more TE gaps are opened at the crossover of electron and hole subbands for more realistic situations. Nevertheless, for weak disorder, the stronger contribution is still observed at the same energies as obtained in the clean limit. 

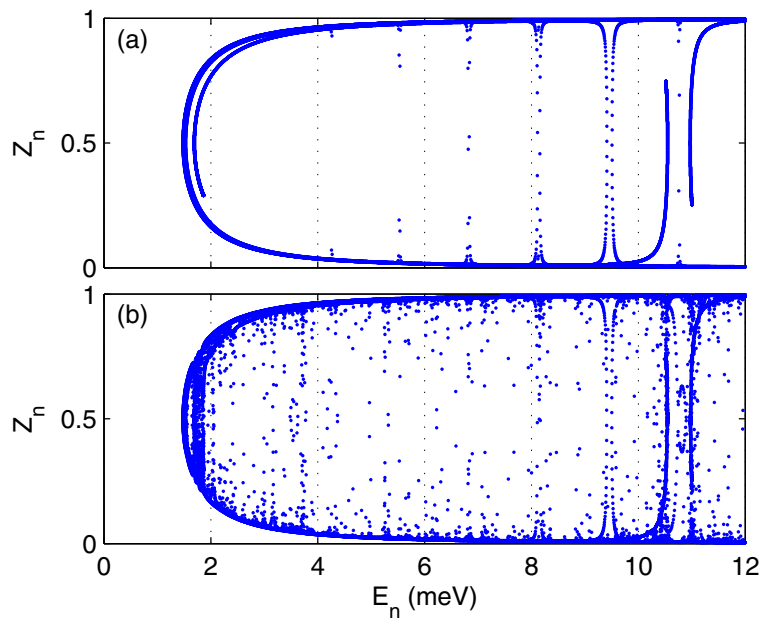

FIG. 5. (Color online) Spectral weight of quasiparticles $Z_{n}$ as a function of energy of states, $E_{n}$, for the nanobelt of width $W=$ $16.8 \mathrm{~nm}(\mathrm{a})$ in the clean limit $(U=0)$ and (b) under the influence of random impurities.

To find the signature of TE, we show the DOS of both superconducting and normal states in the presence of impurities in Fig. 6. The noiselike oscillations in the DOS are imposed over the signature of TE. After matching both oscillations in DOS by mapping the DOS of normal state to a rescale energy range, one can find TE signatures (1) where there are new oscillations in the superconducting DOS, and (2) where there are different oscillatory structures between DOS of superconducting and normal states. In Fig. 6, these signatures are marked by arrows. It is worth noting that the TE modifies the DOS on positive and negative biases symmetrically.

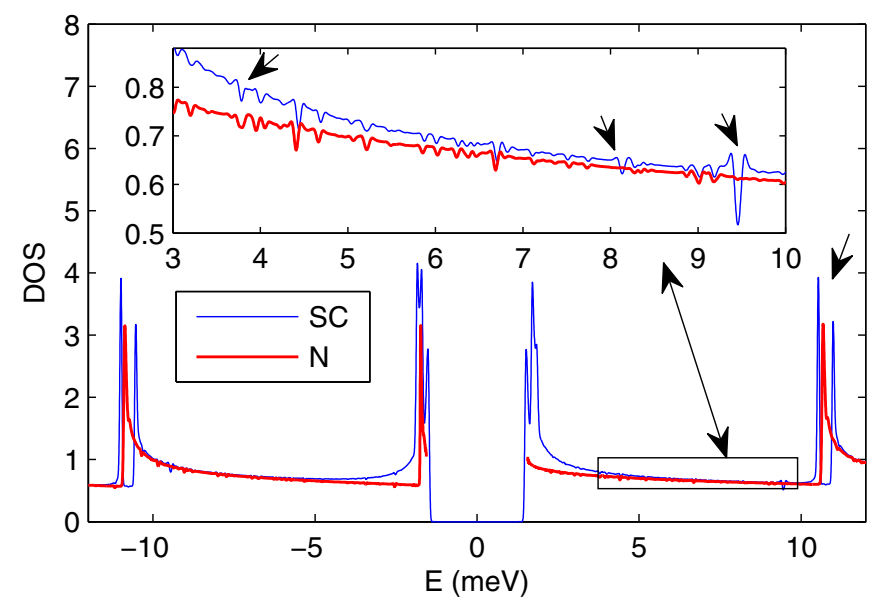

FIG. 6. (Color online) DOS of the superconducting (blue) and normal (red) states for the nanobelt of width $W=16.8 \mathrm{~nm}$ under the influence of the random impurities. The inset shows details of the DOS between $E=3$ and $10 \mathrm{meV}$. The arrows indicate signatures of the TE. Note that the normal DOS is mapped on a new energy scale, in order to match the oscillations in the DOS induced by the impurities. The mapping is done by rescaling, $E_{\text {new }}=\sqrt{E_{\text {old }}^{2}+|\tilde{\Delta}|^{2}}$, where $E_{\text {new }}$ and $E_{\text {old }}$ are the new and old energies, and $\tilde{\Delta}$ is a fitting parameter whose value is close to the mean amplitude of the order parameter $|\bar{\Delta}|$.
Finally, we have to mention that the LDOS under the influence of random impurities shows much more complex patterns. However, the pattern still follows the bonding and antibonding combination of involved quasiparticle wave functions as described in the previous sections.

As the impurity strength increases, the band structure together with order parameter become strongly affected. In this case, while the TE is also strongly enhanced, it becomes increasingly difficult to compare with results obtained in the clean limit. Depending on the particular impurity distribution, TE contributions to the DOS could be individually recognized but these are of different manifestation, when compared to the DOS modifications obtained in the clean limit.

\section{TOMASCH EFFECT IN SUPERCONDUCTING NANOWIRES}

In this section, we consider superconducting nanowires with square and rectangular cross sections. We find a new type of TE, i.e., TE induced by three-subband QPI. Its influence on the electronic structure and its dependence on the symmetry of the system will be discussed in the following sections.

\section{A. Bogoliubov-de Gennes theory for three-dimensional nanowires}

Here, we consider a three-dimensional superconducting nanowire with rectangular cross section (transverse dimensions $L_{x}$ and $L_{y}$ ). Due to quantum confinement in the transverse directions, the Dirichlet boundary conditions are taken on the surface [i.e., $u_{n}(\vec{r})=v_{n}(\vec{r})=0, \vec{r} \in \partial S$ ]. Along the longitudinal direction $z$, we introduce a periodic computational unit cell with length $L_{z}$ where periodic boundary conditions are used.

Due to the fact that the order parameter is independent of the longitudinal direction, i.e., $\Delta(\vec{r})=\Delta(x, y)$, the electronlike and holelike wave functions $u_{n}$ and $v_{n}$ can be expanded, for each longitudinal wave vector $k_{z}$, as

$$
\left(\begin{array}{c}
u_{n}(\vec{r}) \\
v_{n}(\vec{r})
\end{array}\right)=\frac{e^{i k_{z} z}}{\sqrt{L_{z}}} \sum_{j_{x}, j_{y} \in \mathbb{N}^{+}} \phi_{j k}(x, y)\left(\begin{array}{c}
u_{j_{x} j_{y}}^{n} \\
v_{j_{x} j_{y}}^{n}
\end{array}\right),
$$

where

$$
\phi_{j_{x} j_{y}}(x, y)=\frac{2}{\sqrt{L_{x} L_{y}}} \sin \left(\frac{\pi j_{x} x}{L_{x}}\right) \sin \left(\frac{\pi j_{y} y}{L_{y}}\right),
$$

are the eigenstates of the single-electron Schrödinger equation $H_{e} \phi_{j_{x} j_{y}}=\zeta_{j_{x} j_{y}} \phi_{j_{x} j_{y}}$. The longitudinal momentum, $k_{z}$, satisfies the quantization condition, i.e., $k_{z} \cdot L_{z}=2 \pi m, m \in \mathbb{Z}$. Following the previous section, the expansion in Eq. (20) includes the states with energies $-E_{F}<\zeta_{j_{x} j_{y}}<E_{F}+\varepsilon$ where $\varepsilon=$ $5 E_{c}$ is taken sufficiently large in order to guarantee the accuracy.

The pair amplitude $D_{n}(\vec{r})$ and spectral weight $Z_{n}$ for each state are calculated from the Eqs. (4) and (9), respectively. The LDOS $A(\vec{r}, E)$ and the DOS $N(E)$ are calculated from the Eqs. (7) and (8), respectively. 

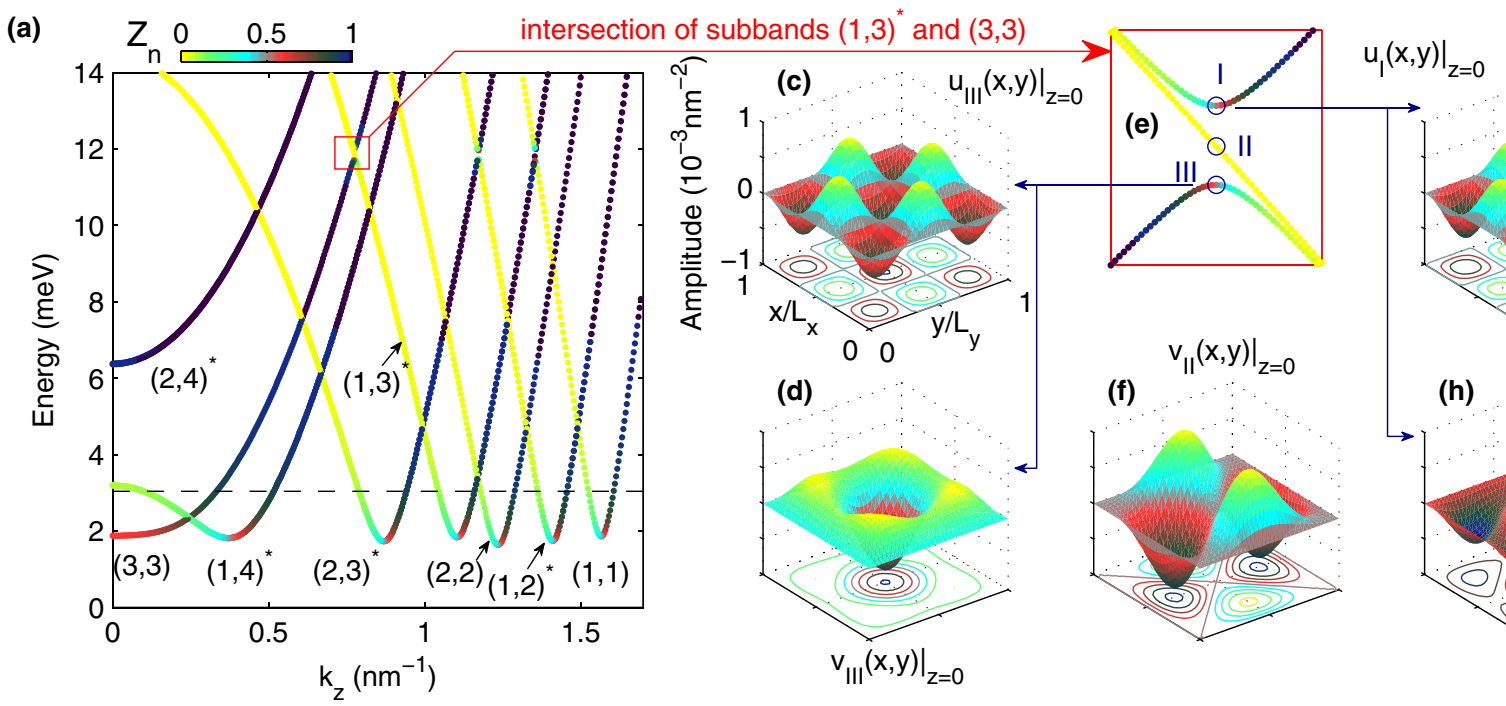

(g)
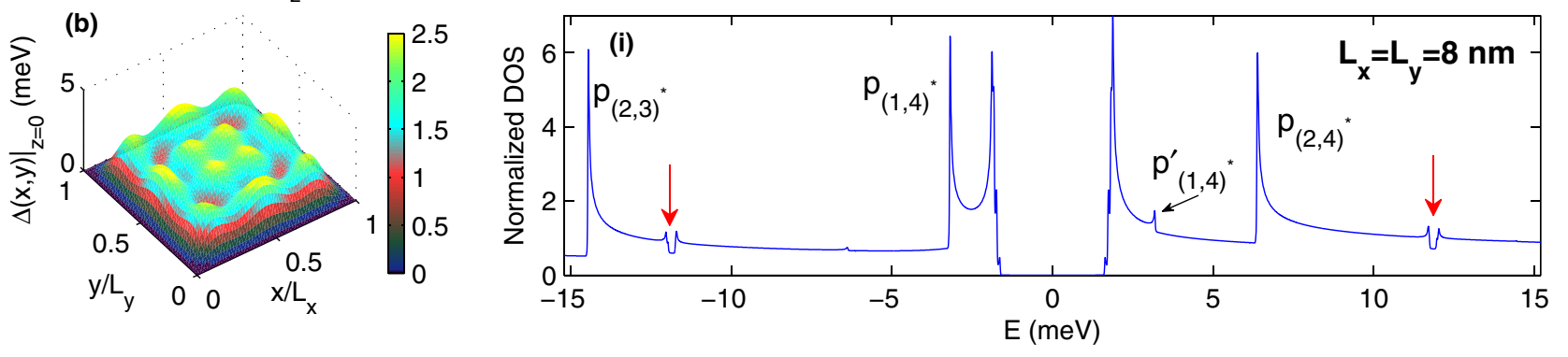

FIG. 7. (Color online) Results for the nanowire with a square cross section: $L_{x}=L_{y}=8 \mathrm{~nm}$. (a) Quasiparticle excitation spectrum as a function of positive longitudinal wave vector $k_{z}$ for the relevant single-electron subbands $\left(j_{x}, j_{y}\right)$. Note that the notation $\left(j_{x}, j_{y}\right)^{*}$ represents two degenerate subbands $\left(j_{x}, j_{y}\right)$ and $\left(j_{y}, j_{x}\right)$. The spectral weight of the quasiparticle states is indicated by color. The excitation spectrum in the red rectangle is magnified in (e) in order to show the influence of the Tomasch effect due to three-subband quasiparticle interference. The three quasiparticle states $I-I I I$ are marked by open circle in (e) and their spatial distribution of electron and hole amplitude, $u$ and $v$, at $z=0$ are shown in (c), (d), (f)-(h). Note that the electron amplitude of the state $I I$ is not shown because $u_{I I}(x, y)=0$. (b) The spatial distribution of the order parameter $\Delta(x, y)$. (i) The corresponding DOS. The gaps due to the Tomasch effect as determined by three-quasiparticle interference processes are marked by red arrows. Peaks $p_{\left(j_{x}, j_{y}\right)}$ are due to the contributions from the states of the bottom of the subbands $\left(j_{x}, j_{y}\right)$, while accompanying peaks $p^{\prime}\left(j_{x}, j_{y}\right)$ are due to the formation of the gaps, i.e., electron-hole coupling.

We use the same microscopic parameters as the one introduced in Sec. II A for bulk $\mathrm{NbSe}_{2}$. The mean electron density $n_{e}=\frac{2}{V} \sum_{n} \int\left\{\left|u_{n}\right|^{2} f\left(E_{n}\right)+\left|v_{n}\right|^{2}\left[1-f\left(E_{n}\right)\right]\right\} d \vec{r}$ for nanowires with $V=L_{x} L_{y} L_{z} . n_{e}$ is kept to its bulk value obtained when $L_{x, y, z} \rightarrow \infty$. All the calculations are performed at zero temperature.

\section{B. Tomasch effect due to three-subband quasiparticles interference}

We first show results in Fig. 7 for a nanowire with square cross section $\left(L_{x}=L_{y}=8 \mathrm{~nm}\right)$ where the geometry of the sample and the order parameter show $C_{4}$ symmetry. Figure 7(a) shows the excitation spectrum as a function of the positive wave vector $k_{z}>0$. All the subbands displayed in the panel are distinguishable and labeled by a set of quantum numbers $\left(j_{x}, j_{y}\right)$. Note that the $\left(j_{x}, j_{y}\right)^{*}$ is a shorthand notation for the pairs $\left(j_{x}, j_{y}\right)$ and $\left(j_{y}, j_{x}\right)$ because of their overlap due to degeneracy. The spectral weight $Z_{n}$ is marked by color for each quasiparticle state.

As seen from Fig. 7(a), the bottom of the subband $(3,3)$ lying below the cutoff energy $E_{c}$ results in a resonant and spatially inhomogeneous order parameter $\Delta(x, y)$, which is shown in Fig. 7(b). $\Delta(x, y)$ shows $C_{4}$ symmetry due to the square cross section. The symmetry of the system and the order parameter determine the properties of TE and its emergence. For example, two sets of TE from two-subband QPI appear at the intersection of subbands $(1,2)^{*}$ and $(1,3)^{*}$, while TE from three-subband QPI appears at the intersection of subbands $(1,3)^{*}$ and $(3,3)$ or $(1,3)^{*}$ and $(1,1)$, respectively.

We next investigate the most significant three-QPI appearing at the intersection of two hole quasiparticle states from subbands $(1,3)^{*}$ and one electron quasiparticle state from subband $(3,3)$. This is indicated by the open red rectangle in Fig. 7(a) and the relevant three dispersion relations of the energy bands are amplified in Fig. 7(e). It is clearly seen that the upper and lower energy bands exhibit a gaplike structure, while the middle energy band crosses the gap diagonally. The Bogoliubov quasiparticles are well formed for the states close to the bottom of the upper band and the top of the lower band. Note that the state pertaining to the middle band are pure holelike quasiparticles states with zero amplitude of the electron component. This is an interesting phenomenon because if a gap opens between states from subbands $(1,3)$ 
and $(3,3)$, the other gap was supposed to be opened between states from subbands $(3,1)$ and $(3,3)$. The reason is that the gap induced by the exchange integral only depends on the symmetry of the wave functions of the relevant states.

In fact, this interesting asymmetrical energy band structure is due to the symmetric and antisymmetric combinations of the two hole states from subbands $(1,3)^{*}$. To see this, in Figs. 7(c), 7(d), and 7(f)-7(h), we show the spatial distribution of the hole and the electron amplitude, $u(x, y)$ and $v(x, y)$, of states $I-I I I$ marked by open circles in Fig. 7(e). The three states have the same wave vector $k_{z}$, chosen such that the gap has a local minimum [see Figs. 7(e)]. The electron components of the gapped states, $u_{I}$ and $u_{I I I}$ [shown in Figs. 7(c) and 7(g)], have contributions only from the electron state of subband $(3,3)$. Therefore they show the same pattern as $\phi_{3,3}$, which is the eigenstate of the single-electron Schrödinger equation with quantum numbers $j_{x}=3$ and $j_{y}=3$. For the corresponding amplitude of hole components, $v_{I}$ and $v_{I I I}$ [shown in Figs. 7(d) and $7(\mathrm{~h})]$, they have the same spatial distribution as the symmetric combination of the two eigenstates, i.e., $\phi_{1,3}+\phi_{3,1}$, but with opposite sign for the two amplitudes $I$ and $I I I$.

Clearly, the Bogoliubov quasiparticle states, I and II I, are the bonding and antibonding combinations of the electron and hole components. The reason is that both the wave-functions and the order parameter exhibit $C_{4}$ symmetry and, thus, result in nonzero exchange integrals, which are responsible for TE and generate energy gaps. The quasiparticle state II does not take part in the quasiparticle interference because its hole component, $v_{I I}$, has $C_{2}$ symmetry, $\left[v_{I I}(x, y)=-v_{I I}(y, x)\right]$, due to the antisymmetric combination of the two hole eigenstates, i.e., $\phi_{1,3}-\phi_{3,1}$, leading to a vanishing exchange integral.

We now conclude the appearance of TE due to threesubband QPI. First, hole states from subbands $(1,3)$ and $(3,1)$ are combined in order to generate symmetric and antisymmetric states but which are degenerate in energy. Then, the symmetric combination couples with the electron state from subband $(3,3)$ and forms Bogoliubov quasiparticle states, therefore inducing a gap. Finally, the energy and the wave function of the antisymmetric combination is unaffected.

The process results in oscillations in the DOS, which are symmetrical in bias [see Fig. 7(i)]. The oscillations induced by TE from the three-QPI are marked by arrows for both positive and negative biases. When comparing with the DOS of a nanobelt shown in Fig. 1(h), we notice that there are less oscillations induced by TE. The reason is that TE emerges only in case of a nonzero exchange integral. This becomes harder to achieve for a system with two quantum numbers, $\left(j_{x}, j_{y}\right)$, because the condition has to be fulfilled by both. Next we will show that the TE of three-subband QPI in a nanowire depends strongly on the symmetry of the electronic structures and the geometry of the sample. For this purpose, we consider nanowires with rectangular cross section, i.e., $L_{x} \neq L_{y}$ where the $C_{4}$ symmetry is broken and, more importantly, results in the splitting of the subbands $\left(j_{x}, j_{y}\right)$ and $\left(j_{y}, j_{x}\right)$. In Fig. 8, we present the appearance of the TE due to three-subband QPI for states of hole subbands $(1,3),(3,1)$ and electron subband $(3,3)$ for nanowires with $L_{x}=8 \mathrm{~nm}$ and $L_{y}=8.03,8.05,8.07$, and $8.10 \mathrm{~nm}$, respectively. The spectral weight of the relevant states is indicated by color. The three subbands without TE are shown

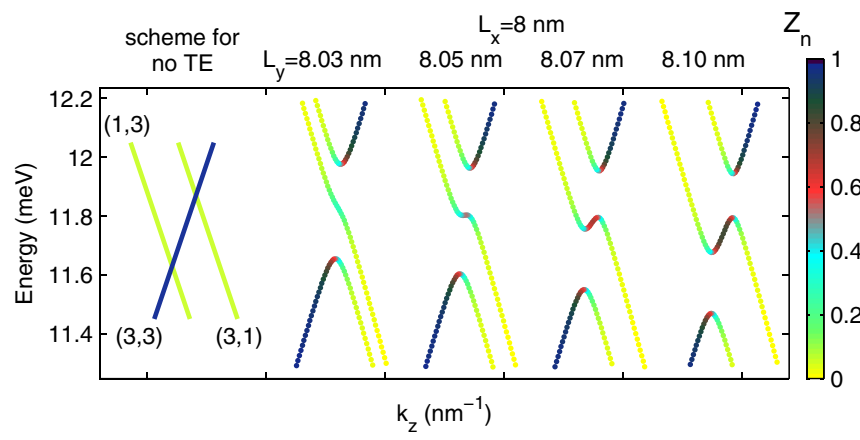

FIG. 8. (Color online) Symmetry-dependent Tomasch effect of three-subband quasiparticles interference for states of subbands $(1,3)$, $(3,1)$, and $(3,3)$ for rectangular cross section $L_{x}<L_{y}$. The left-most panel shows the band dispersion for the three subbands without the Tomasch effect. The other four panels show the band dispersion versus longitudinal wave vector $k_{z}$ for $L_{x}=8 \mathrm{~nm}$ and $L_{y}=8.03,8.05,8.07$, and $8.10 \mathrm{~nm}$, respectively. They are shifted horizontally for clarity. The spectral weight of the quasiparticle states is indicated by color in all panels.

in the most left panel. As mentioned previously, the two hole subbands $(1,3)$ and $(3,1)$ split while the electron subband $(3,3)$ crosses them. The states of subband $(3,1)$ have higher energy than those of subband $(1,3)$ for a given $k_{z}$ when $L_{y}>L_{x}$. It can be seen that the bottom of the highest energy subband shifts to the right with increasing $L_{y}$, while the top of the lowest energy subband shifts to the left. When comparing with the result for a square cross section shown in Fig. 7(e), we find that the previously unaffected hole subband becomes and shows mixed electron-hole components, signaling a coupling with the electron subband. Finally, the three-subband QPI converts into two sets of two-subband QPI, appearing for states from

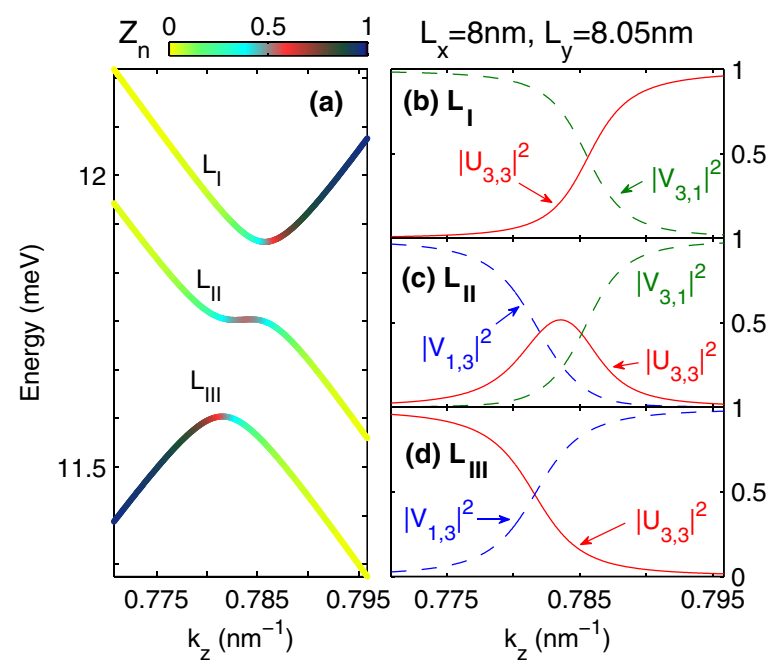

FIG. 9. (Color online) (a) Band dispersion for the energy bands $\left(L_{I}-L_{I I I}\right)$ due to the Tomasch effect of three-quasiparticles interference of the energy subbands $(1,3),(3,1)$, with $(3,3)$ for a rectangular cross section $L_{x}=8 \mathrm{~nm}$ and $L_{y}=8.05 \mathrm{~nm}$. The spectral weight of the quasiparticle states are indicated by color. The electron and hole components of these states are shown in (b)-(d), respectively. 
subbands $(1,3)$ and $(3,3)$ and separately from subbands $(3,1)$ and $(3,3)$, as seen in Fig. 8 for $L_{y}=8.1 \mathrm{~nm}$.

An interesting phenomenon is noticed for $L_{y}=8.05 \mathrm{~nm}$ where the middle energy band exhibits a flat region, as seen in Fig. 8. The spectral weight $Z_{n}$ and the corresponding states [see Fig. 9] show that the Bogoliubov quasiparticle states couple the hole and electron components. For the upper energy band $L_{I}$, the quasiparticle states are converted from the hole-like states $v_{3,1}$ to the electron-like states $u_{3,3}$ as $k_{z}$ is increased, as seen in Fig. 9(b). The same is true for the lower energy band $L_{I I I}$ but now from the electronlike states $u_{3,3}$ to the hole-like states $v_{1,3}$, as seen in Fig. 9(d). The middle energy band shows a more complex coupling among the three subbands as it is converted from $v_{1,3}$ to $v_{3,1}$ with the help of $u_{3,3}$ due to the compatible symmetry of these states.

\section{CONCLUSION AND DISCUSSION}

In conclusion, we investigated the Tomasch effect on the electronic structure in nanoscale superconductors by solving the Bogoliubov-de Gennes equations self-consistently. Here, the Tomasch effect is induced by an inhomogeneous order parameter appearing due to quantum confinement. We found that the Tomasch effect couples degenerate electron and hole states above the superconducting gap due to quasiparticle interference leading to additional pairs of BCS-like Bogoliubov-quasiparticles that generate energy gaps resulting in oscillations in the DOS. When the energies of the paired states are far from the Fermi level, the pair states show pseudo-gap-like structures in the DOS. When they are close to the Fermi level, the pair states result in modulated wave patterns in the local density of states. All these are due to the intersubband electron-hole coupling and their bonding and antibonding combinations generating the pair states.

The Tomasch effect is strongly related to the geometrical symmetry of the system and the symmetry, parity and spacial variation of the order parameter. For the nanobelt, the Tomasch effect only leads to two-subband quasiparticles interference processes. With even-parity order parameter in the clean limit, the Tomasch effect only plays a role for two states with the same parity. A reduced $4 \times 4 \mathrm{BdG}$ matrix can describe well the results. For a nanowire with a square cross section, the Tomasch effect also results in three-subband quasiparticle interference processes due to the higher degree of symmetry. We observe coupling only for the symmetric combination of two hole states, while the antisymmetric one remains unaffected. This leads to a unique energy band structure, where one of the subband crosses diagonally across the induced gap. For nanowires with rectangular cross section, the three-subband quasiparticles interference is converted into two sets of two-subband quasiparticles interference leading to a distortion of the previously unaffected band.

The Tomasch effect is commonly formed in inhomogeneous superconductivity but it could be difficult to observe it experimentally. One reason is that the effect can be shadowed by other states present at the same energy. Another reason is that the large size of Cooper pairs may result in a complex global electronic structure. However, the effect can be enhanced in the following ways: (1) by reducing the symmetry of the sample such as realized by surface roughness and by making layered junctions; (2) by breaking the symmetry of the order parameter by, e.g., disorder and impurities, and (3) by designing the sample such that the relevant electron and hole subbands touch each other near their bottom and top, respectively. We have show that for a realistic case, in the presence of weak disorder, the modifications in the DOS due to the TE survive and can be clearly distinguished from oscillations induced by impurity scattering.

\section{ACKNOWLEDGMENT}

This work was supported by the Flemish Science Foundation (FWO-Vlaanderen) and the Methusalem funding of the Flemish Government.
[1] K. von Klitzing, Rev. Mod. Phys. 58, 519 (1986).

[2] X.-L. Qi and S.-C. Zhang, Rev. Mod. Phys. 83, 1057 (2011).

[3] P. G. De Gennes, Superconductivity of Metals and Alloys (Westview Press, Reading, MA, 1999).

[4] J. Bardeen, L. N. Cooper, and J. R. Schrieffer, Phys. Rev. 108, 1175 (1957).

[5] C. Caroli, P. G. De Gennes, and J. Matricon, Phys. Lett. 9, 307 (1964).

[6] J. Bardeen, R. Kümmel, A. E. Jacobs, and L. Tewordt, Phys. Rev. 187, 556 (1969).

[7] F. Gygi and M. Schlüter, Phys. Rev. B 43, 7609 (1991).

[8] N. Hayashi, T. Isoshima, M. Ichioka, and K. Machida, Phys. Rev. Lett. 80, 2921 (1998)

[9] S. M. M. Virtanen and M. M. Salomaa, Phys. Rev. B 60, 14581 (1999).

[10] K. Tanaka, I. Robel, and B. Janko, Proc. Natl. Acad. Sci. USA 99, 5233 (2002).
[11] A. S. Mel'nikov, D. A. Ryzhov, and M. A. Silaev, Phys. Rev. B 79, 134521 (2009).

[12] H. F. Hess, R. B. Robinson, and J. V. Waszczak, Phys. Rev. Lett. 64, 2711 (1990).

[13] T. Nishio, T. An, A. Nomura, K. Miyachi, T. Eguchi, H. Sakata, S. Lin, N. Hayashi, N. Nakai, M. Machida, and Y. Hasegawa, Phys. Rev. Lett. 101, 167001 (2008).

[14] T. Cren, D. Fokin, F. Debontridder, V. Dubost, and D. Roditchev, Phys. Rev. Lett. 102, 127005 (2009).

[15] T. Cren, L. Serrier-Garcia, F. Debontridder, and D. Roditchev, Phys. Rev. Lett. 107, 097202 (2011).

[16] S. Bose, A. M. Garcia-Garcia, M. M. Ugeda, J. D. Urbina, C. H. Michaelis, I. Brihuega, and K. Kern, Nat. Mater. 9, 550 (2010).

[17] Y. Guo, Y.-F. Zhang, X.-Y. Bao, T.-Z. Han, Z. Tang, L.-X. Zhang, W.-G. Zhu, E. G. Wang, Q. Niu, Z. Q. Qiu, J.-F. Jia, Z.-X. Zhao, and Q.-K. Xue, Science 306, 1915 (2004).

[18] S. Qin, J. Kim, Q. Niu, and C.-K. Shih, Science 324, 1314 (2009). 
[19] A. A. Shanenko, M. D. Croitoru, and F. M. Peeters, Phys. Rev. B 75, 014519 (2007).

[20] M. D. Croitoru, A. A. Shanenko, and F. M. Peeters, Phys. Rev. B 76, 024511 (2007).

[21] A. A. Shanenko, M. D. Croitoru, A. V. Vagov, V. M. Axt, A. Perali, and F. M. Peeters, Phys. Rev. A 86, 033612 (2012).

[22] A. A. Shanenko, M. D. Croitoru, and F. M. Peeters, Phys. Rev. B 78, 024505 (2008).

[23] Y. Mizohata, M. Ichioka, and K. Machida, Phys. Rev. B 87, 014505 (2013).

[24] A. A. Shanenko, M. D. Croitoru, R. G. Mints, and F. M. Peeters, Phys. Rev. Lett. 99, 067007 (2007).

[25] L.-F. Zhang, L. Covaci, M. V. Milošević, G. R. Berdiyorov, and F. M. Peeters, Phys. Rev. Lett. 109, 107001 (2012); Phys. Rev. B 88, 144501 (2013).

[26] L.-F. Zhang, L. Covaci, and F. M. Peeters, arXiv:1401.4319 [cond-mat].

[27] W. J. Tomasch, Phys. Rev. Lett. 15, 672 (1965).

[28] W. J. Tomasch, Phys. Rev. Lett. 16, 16 (1966).

[29] W. L. McMillan and P. W. Anderson, Phys. Rev. Lett. 16, 85 (1966).
[30] T. Wolfram, Phys. Rev. 170, 481 (1968).

[31] O. Nesher and G. Koren, Appl. Phys. Lett. 74, 3392 (1999).

[32] B. P. Stojković and O. T. Valls, Phys. Rev. B 50, 3374 (1994).

[33] B. P. Stojković and O. T. Valls, Phys. Rev. B 49, 3413 (1994).

[34] J. E. Hoffman, K. McElroy, D.-H. Lee, K. M. Lang, H. Eisaki, S. Uchida, and J. C. Davis, Science 297, 1148 (2002).

[35] T. Hanaguri, Y. Kohsaka, J. C. Davis, C. Lupien, I. Yamada, M. Azuma, M. Takano, K. Ohishi, M. Ono, and H. Takagi, Nat. Phys. 3, 865 (2007).

[36] T. Hänke, S. Sykora, R. Schlegel, D. Baumann, L. Harnagea, S. Wurmehl, M. Daghofer, B. Büchner, J. van den Brink, and C. Hess, Phys. Rev. Lett. 108, 127001 (2012).

[37] J.-X. Zhu, K. McElroy, J. Lee, T. P. Devereaux, Q. Si, J. C. Davis, and A. V. Balatsky, Phys. Rev. Lett. 97, 177001 (2006).

[38] C. N. Lau, N. Markovic, M. Bockrath, A. Bezryadin, and M. Tinkham, Phys. Rev. Lett. 87, 217003 (2001).

[39] K. Y. Arutyunov, D. S. Golubev, and A. D. Zaikin, Phys. Rep. 464, 1 (2008).

[40] N. C. Koshnick, H. Bluhm, M. E. Huber, and K. A. Moler, Science 318, 1440 (2007).

[41] K. Y. Arutyunov, T. T. Hongisto, J. S. Lehtinen, L. I. Leino, and A. L. Vasiliev, Sci. Rep. 2, 293 (2012). 